\title{
Preoperative Chemotherapy plus Lapatinib or Trastuzumab or Both in HER2-Positive Operable Breast Cancer (CHERLOB Trial)
}

\author{
Valentina Guarneri,1 Antonio Frassoldati,1 Federico Piacentini,1 \\ Gordana Jovic,1 Simona Giovannelli,1 Cristina Oliva,2 \\ PierFranco Conte1
}

Clinical Breast Cancer, Vol. 8, No. 2, 192-194, 2008

Key words: Epidermal growth factor receptor, Immunohistochemistry, Pathologic complete response

\section{Rationale}

Primary systemic therapy is the standard of care for locally advanced and inflammatory breast carcinoma, and is progressively more applied also in earlier stages of disease. Nowadays, this strategy can be considered a reasonable alternative to postoperative therapy for all patients who are candidates for adjuvant chemotherapy.

In patients with HER2-positive tumors, several trials evaluating adjuvant trastuzumab have shown a clear advantage compared with chemotherapy alone. ${ }^{1-4}$ Therefore, trastuzumab represents an essential component of the adjuvant treatment of HER2-positive breast cancer.

In the preoperative setting, the combination of trastuzumab to sequential chemotherapy with taxanes and anthracyclines resulted in an impressive rate of pathologic complete responses (pCRs), 5 which represents a powerful marker of long-term outcome6,7. However, not all the patients equally benefit from trastuzumab therapy, and many different intracellular pathways can contribute to the intrinsic or acquired resistance to HER2 blockade. ${ }^{8}$ Lapatinib is a smallmolecule dual inhibitor of the tyrosine kinase activity of epidermal growth factor receptor (EGFR) and HER2. The inhibition of these two pathways can affect tumor growth

EUDRACT number: 2006-001839-21

NCI ClinicalTrials.gov number: NCT0042299

${ }^{1}$ Division of Medical Oncology, Department of Oncology and Hematology, University Hospital, University of Modena and Reggio Emilia, Modena, Italy

${ }^{2}$ Oncology Medicine Development Center, GlaxoSmithKline, Greenford, United Kingdom

Submitted: Apr 26, 2007; Revised: Jul 31, 2007; Accepted: Aug 22, 2007

Address for correspondence: PierFranco Conte, MD, Department of Oncology and Hematology, University Hospital, via del Pozzo 71,41100 Modena, Italy

Fax: 39-059-422-4429; e-mail: conte.pierfranco@unimore.it by reducing the EGFR-dependent proliferative stimulus, by restoring apoptosis, and possibly by enhancing sensitivity to chemotherapy.9,10 However, clinical data so far support the activity of lapatinib in patients with HER2-positive/amplified tumors only. ${ }^{11}$ Furthermore, this agent can also act against tumoral cells that express the truncated form of EGFR and HER2, which are not recognized by antibodies directed to the external domain. ${ }^{12}$ In metastatic disease, the combination of lapatinib and capecitabine significantly improved the progression-free survival compared with capecitabine alone in patients pretreated with trastuzumab.13 Therefore, lapatinib in combination wtih capecitabine has recently been approved for patients with HER2-positive metastatic breast cancer who have progressed on previous therapy including trastuzumab. The combination trastuzumab/lapatinib is also under development because of the potential additive effect of a combined blockade of the outer and inner part of the HER2 receptor; preliminary safety and activity data seem promising. ${ }^{14}$

On these premises, the combination of lapatinib with chemotherapy and trastuzumab represents an attractive research tool. Therefore, we have designed a phase II randomized trial to evaluate the activity and safety of chemotherapy plus lapatinib, trastuzumab, or both trastuzumab and lapatinib as preoperative therapy for HER2-positive operable breast cancer.

\section{EGF109085-LAP106988/CHERLOB Trial}

This is a phase IIb randomized trial in which patients with HER2-positive primary breast cancer stage II-IIIA (tumor size $>2 \mathrm{~cm}$ ) are randomized to receive chemotherapy plus trastuzumab (arm A), or chemotherapy plus lapatinib (arm B), or chemotherapy plus trastuzumab/lapatinib (arm C). The primary endpoint of this study is the percentage of $\mathrm{pCR}$, defined as complete disappearance of invasive tumor in breast and axillary lymph nodes. Secondary aims are as follows: the percentage of clinical objective responses in the breast; the

Electronic forwarding or copying is a violation of US and International Copyright Laws.

Authorization to photocopy items for internal or personal use, or the internal or personal use of specific clients, is granted by CIG Media Group, LP

ISSN \#1526-8209, provided the appropriate fee is paid directly to Copyright Clearance Center, 222 Rosewood Drive, Danvers, MA 01923 USA 978-750-8400. 


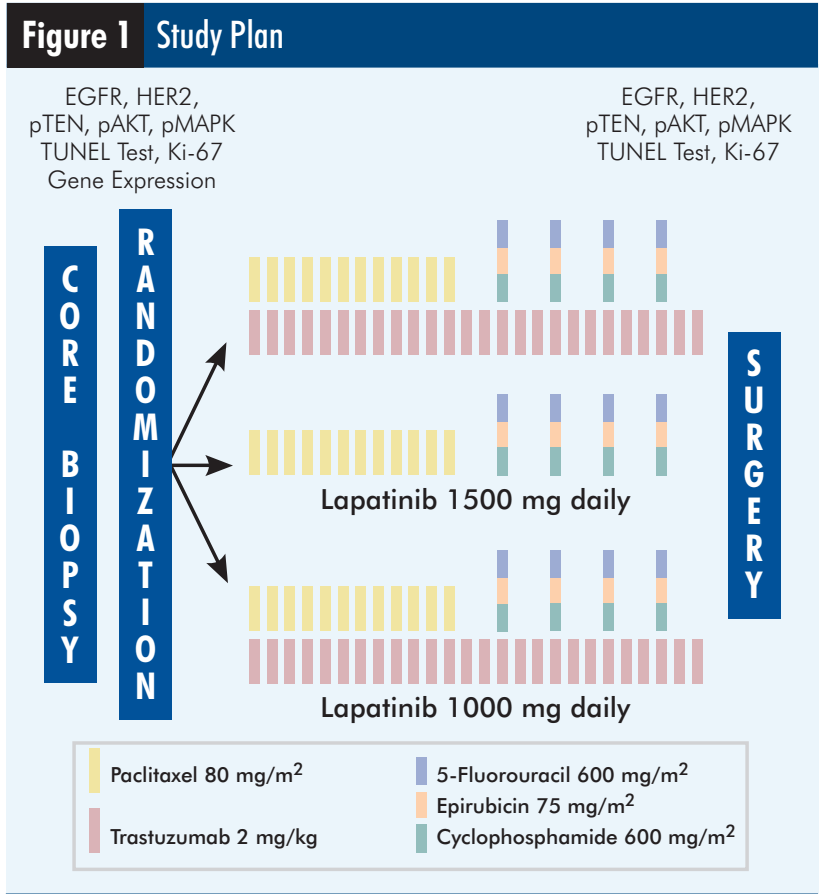

percentage of conservative surgery; the safety profile of these combinations; the time to treatment failure from start of primary therapy; the percentage of inhibition of intermediate and final biomarkers of the proliferative and the apoptosis pathways induced by the different combinations; and the correlation between gene expression at diagnosis and pathologic response. This trial is currently ongoing in several institutions in Europe. The local human investigation committees of participating institutions approved the protocol.

\section{Inclusion and Exclusion Criteria}

Patients are eligible for this trial if they met the following criteria: previously untreated, infiltrating primary breast cancer of $>2 \mathrm{~cm}$ in largest clinical diameter; HER2 positivity (immunohistochemistry [IHC] $3+$ or fluorescence in situ hybridization+); age between 18 and 65 years; Eastern Cooperative Oncology Group performance status of $0 / 1$; availability of tumor tissue suitable for biologic and molecular examination before starting primary treatment; left ventricular ejection fraction (LVEF) within the institutional range of normal; normal organ and marrow function; ability to swallow and retain oral medication; and provision of written informed consent. Key exclusion criteria are as follows: stage IIIB, IIIC, and inflammatory breast cancer; presence of distant metastases; uncontrolled intercurrent illness including, but not limited to, ongoing or active infection, symptomatic congestive heart failure, unstable angina pectoris, cardiac arrhythmia, or psychiatric illness/social situations that would limit compliance with study requirements; pregnancy or breastfeeding; women of childbearing potential who refuse to adopt adequate contraceptive measures; patients HIV-positive who are receiving combination antiretroviral
Table 1 Rules for Treatment Discontinuation in Case of Asymptomatic Cardiac Events

\begin{tabular}{l|c|c|c}
$\begin{array}{c}\text { Relationship of } \\
\text { LVEF to LLN }\end{array}$ & $\begin{array}{c}\text { Absolute } \\
\text { Decrease } \\
\text { of }<10 \%\end{array}$ & $\begin{array}{c}\text { Absolute } \\
\text { Decrease } \\
\text { of } 10 \%-15 \%\end{array}$ & $\begin{array}{c}\text { Absolute } \\
\text { Decrease } \\
\text { of } \geq 16 \%\end{array}$ \\
\hline $\begin{array}{l}\text { Within the } \\
\text { Normal Limits }\end{array}$ & Continue & Continue & Hold ${ }^{\dagger}$ \\
\hline $\begin{array}{l}1 \%-5 \%<\text { LLN } \\
\geq 6 \%<\text { LLN }\end{array}$ & Continue & Hold ${ }^{\dagger}$ & Hold ${ }^{\dagger}$ \\
\hline
\end{tabular}

*Reassessment after 4 weeks (and every 4 weeks thereafter).

$\uparrow$ Arm A (chemotherapy plus trastuzumab): stop trastuzumab. Reassess after 4 weeks. If criteria for continuation is met, resume treatment. If criteria for continuation is unmet, reassess after 4 additional weeks. If criteria for continuation met, resume treatment. If there is no recovery after 8 weeks of holding trastuzumab, discontinue trastuzumab. Arm B (chemotherapy plus lapatinib): stop lapatinib. Reassess after 1 week. If LVEF is above lower limit of normal, resume lapatinib full dose. If LVEF is below lower limit of normal, repeat LVEF assessment after additional week. If LVEF is above lower limit of normal, restart at reduced dose $(1250 \mathrm{mg})$. If there is no recovery after 2 weeks of holding lapatinib, discontinue lapatinib. Arm C (chemotherapy plus trastuzumab/lapatinib). Stop trastuzumab and lapatinib. Reassess after 1 week. If LVEF is above lower limit of normal, resume lapatinib full dose. Reassess LVEF 4 weeks from trastuzumab discontinuation. If LVEF is above lower limit of normal, resume trastuzumab. If LVEF is below lower limit of normal, repeat LVEF assessment after additional week. If LVEF is above lower limit of normal, restart lapatinib at reduced dose $(750 \mathrm{mg})$. Reassess LVEF 4 weeks from trastuzumab discontinuation. If LVEF is above lower limit of normal, resume trastuzumab. If there is no recovery after 2 weeks of holding lapatinib and/or 8 weeks of holding trastuzumab, discontinue lapatinib and/or trastuzumab.

In case of restart of treatment after any discontinuation because of LVEF abnormalities, LVEF should be assessed every 4 weeks until surgery.

Abbreviation: $L L N=$ lower limit of normal

therapy; gastrointestinal (GI) tract disease resulting in an inability to take oral medication, malabsorption syndrome, a requirement for intravenous alimentation, previous surgical procedures affecting absorption, uncontrolled inflammatory GI disease; and concomitant requirement for medication classified as CYP3A4 inducers or inhibitors.

\section{Sample Size}

The sample size has been estimated by using the 2-stage Simon's design. We assumed that the expected rate of pCR with chemotherapy will be $20 \%$. We will consider worthwhile the combination of chemotherapy plus trastuzumab ( $\operatorname{arm~A}$ ) or plus lapatinib (arm B) if the incidence of $\mathrm{pCR}$ will be equal to $40 \%$. Moreover, we will consider worthwhile the combination of chemotherapy plus trastuzumab/lapatinib (arm C) if the rate of $\mathrm{pCR}$ will be equal to $60 \%$. With $\alpha=10 \%, \beta=10 \%$, the following sample sizes have been obtained: arm A and arm B: first stage 17 patients; if $\geq 4$ pCRs are observed, 20 additional patients per arm will be recruited for a total of 37 patients in each arm; arm C: first stage 18 patients; if $\geq 8$ pCRs are observed, 28 additional patients will be recruited for a total of 46 patients. Overall, if the hypothesis is fulfilled, a total of 120 patients will be enrolled in the 3 arms.

\section{Trial Design}

Patients randomized to arm A receive chemotherapy with weekly paclitaxel $80 \mathrm{mg} / \mathrm{m}^{2}$ for 12 weeks followed by 4 courses of FEC (5-fluorouracil [5-FU] $600 \mathrm{mg} / \mathrm{m}^{2}$, epirubicin $75 \mathrm{mg} / \mathrm{m}^{2}$, cyclophosphamide $600 \mathrm{mg} / \mathrm{m}^{2}$ ) administered every 
3 weeks plus trastuzumab $4 \mathrm{mg} / \mathrm{kg}$ loading dose followed by $2 \mathrm{mg} / \mathrm{kg}$ weekly for the whole duration of treatment (26 weeks). Patients randomized to arm B receive the same chemotherapy regimen plus lapatinib $1500 \mathrm{mg}$ orally daily for the whole duration of chemotherapy. Patients randomized to arm $\mathrm{C}$ receive the same chemotherapy regimen plus trastuzumab $2 \mathrm{mg} / \mathrm{kg}$ weekly (fisrt loading dose $4 \mathrm{mg} / \mathrm{kg}$ ) plus lapatinib $1000 \mathrm{mg}$ orally daily for the whole treatment period (Figure 1). Surgery is planned within 2 weeks since the last trastuzumab/lapatinib dose.

\section{Monitoring of Cardiac Safety}

Only patients with LVEF within the institutional limits of normal at baseline as measured by either echocardiography or multigated radionucleotide angiography scan are eligible for this protocol. Left ventricular ejection fraction evaluation is repeated after 12 weeks (before start of the FEC regimen) and at the completion of treatment. More frequent assessment of LVEF should be repeated in any patients with signs or symptoms suspicious of cardiac failure. The rules for the management of asymptomatic decline in the LVEF are summarized in Table 1. A > 16\% absolute decrease from baseline in LVEF (asymptomatic or symptomatic), that is below the institution's lower limit of normal is considered a serious adverse event.

\section{Gene Expression Profile and Biomarker Evaluation}

To define the inhibition of the downstream pathways of the EGFR family, the following biomarkers are centrally evaluated: EGFR, HER2; pTEN, pAKT, pMAPK; apoptosis (TUNEL test); and Ki-67. These parameters are evaluated by IHC on paraffin-embedded specimens obtained from diagnostic core biopsy of the primary lesion and from surgical specimens. Moreover, fresh tumor tissue from the diagnostic core-biopsy is collected and snap frozen to perform a microarray analysis of the gene expression profile before treatment and to evaluate its correlation with response.

\section{Conclusion}

This is the first trial exploring the combination of anthracycline-based chemotherapy plus trastuzumab, lapatinib, or both as primary systemic therapy for patients with HER2positive operable breast cancer. This trial will hopefully provide very useful information for optimizing the use of anti-HER2 agents in early breast cancer.

\section{References}

1. Slamon D, Eiermann W, Robert N, et al. Phase III randomized trial comparing doxorubicin and cyclophosphamide followed by docetaxel (ACT) with doxorubicin and cyclophosphamide followed by docetaxel and trastuzumab (ACTH) with docetaxel, carboplatin and trastuzumab (TCH) in HER2 positive early breast cancer patients: BCIRG 006 study. Presented at: the 28th Annual San Antonio Breast Cancer Symposium; December 8-11, 2005; San Antonio, TX. Abstract 1.

2. Romond EH, Perez EA, Bryant J, et al. Trastuzumab plus adjuvant chemotherapy for operable HER2-positive breast cancer. $N$ Engl $J$ Med 2005; 353:1673-84.

3. Piccart-Gebhart MJ, Procter M, Leyland-Jones B, et al. Trastuzumab after adjuvant chemotherapy in HER2-positive breast cancer. $N$ Engl J Med 2005; 353:1659-72.

4. Joensuu H, Kellokumpu-Lehtinen PL, Bono P, et al. Adjuvant docetaxel or vinorelbine with or without trastuzumab for breast cancer. $N$ Engl J Med 2006; 354:809-20.

5. Buzdar AU, Ibrahim NK, Francis D, et al. Significantly higher pathologic complete remission rate after neoadjuvant therapy with trastuzumab, paclitaxel, and epirubicin chemotherapy: results of a randomized trial in human epidermal growth factor receptor 2-positive operable breast cancer. J Clin Oncol 2005; 23:3676-85.

6. Wolmark N, Wang J, Mamounas E, et al. Preoperative chemotherapy in patients with operable breast cancer: nine-year results from National Surgical Adjuvant Breast and Bowel Project B-18. J Natl Cancer Inst Monogr 2001; 30:96-102.

7. Guarneri V, Broglio K, Kau SW, et al. Prognostic value of pathologic complete response after primary chemotherapy in relation to hormone receptor status and other factors. J Clin Oncol 2006; 24:1037-44.

8. Nahata R, Esteva F. Molecular mechanism of trastuzumab resistance. Breast Cancer Res 2006; 8:215.

9. Konecny GE, Pegram MD, Venkatesan N, et al. Activity of the dual kinase inhibitor lapatinib (GW572016) against HER-2-overexpressing and trastuzumab-treated breast cancer cells. Cancer Res 2006; 66:1630-9.

10. Spector NL, Xia W, Burris H III, et al. Study of the biological effects of lapatinib, a reversible inhibitor of ErbB1 and ErbB2 tyrosine kinases, on tumor growth and survival pathways in patients with advanced malignancies. J Clin Oncol 2005; 23:2502-12.

11. Finn RS, Press MF, Dering J, et al. Biomarker analysis of lapatinib with paclitaxel versus paclitaxel as first-line treatment in 579 patients with metastatic breast cancer. Presented at: the 14th European Cancer Conference; September 23-27, 2007; Barcelona, Spain. Abstract 2097.

12. Xia W, Liu LH, Ho P, et al. Truncated ErbB2 receptor (p95ErbB2) is regulated by heregulin through heterodimer formation with ErbB3 yet remains sensitive to the dual EGFR/ErbB2 kinase inhibitor GW572016. Oncogene 2004; 23:646-53.

13. Geyer CE, Forster J, Lindquist D, et al. Lapatinib plus capecitabine for HER2-positive advanced breast cancer. $N$ Engl J Med 2006; 355:273343.

14. Storniolo AM, Burris H, Pegram M, et al. A phase I, open-label study of lapatinib (GW572016) plus trastuzumab; a clinically active regimen. $J$ Clin Oncol 2005, 23(16 suppl):18s (Abstract 559). 